\title{
Vortex liquid in the superconducting vacuum of the quenched QCD induced by strong magnetic field*
}

\section{V. Braguta}

IHEP, Protvino, Moscow region, 142284 Russia

ITEP, B. Cheremushkinskaya str. 25, Moscow, 117218 Russia

MIPT, Institutskii per. 9, Dolgoprudny, Moscow Region, 141700 Russia

\section{P. V. Buividovich}

Institute of Theoretical Physics, University of Regensburg, Universitätsstrasse 31, D-93053

Regensburg, Germany

\section{N. Chernodub}

CNRS, Laboratoire de Mathématiques et Physique Théorique, Université François-Rabelais Tours, Parc de Grandmont, 37200 Tours, France

Department of Physics and Astronomy, University of Gent, Krijgslaan 281, S9, B-9000 Gent, Belgium

\section{A. Yu. Kotov}

ITEP, B. Cheremushkinskaya str. 25, Moscow, 117218 Russia

\section{I. Polikarpov}

ITEP, B. Cheremushkinskaya str. 25, Moscow, 117218 Russia

MIPT, Institutskii per. 9, Dolgoprudny, Moscow Region, 141700 Russia

In the background of the strong magnetic field the vacuum is suggested to possess an electromagnetically superconducting phase characterised by the charged $\rho$-meson condensate. The $\rho$-meson condensate is inhomogeneous due to the presence of the string-like defects which are parallel to the magnetic field. In agreement with these expectations, we have observed the presence of the $\rho$-vortices in numerical simulations of the vacuum of the quenched two-color lattice QCD in strong magnetic field background. We have found that in the quenched QCD the $\rho$-vortices form a liquid.

31st International Symposium on Lattice Field Theory - LATTICE 2013

July 29 - August 03, 2013

Mainz, Germany

${ }^{*}$ The work was partially supported by grant No. ANR-10-JCJC-0408 HYPERMAG (France). Numerical calculations were performed at the ITEP system Stakan. There is an ongoing work with M. Mueller-Preussker on studying the problem with dynamical quarks.

†n leave from ITEP, Moscow, Russia.

¥Speaker. 


\section{Introduction}

Recently it was suggested that in an external magnetic field the vacuum of QCD becomes a superconductor if the strength of the magnetic field exceeds the critical value [1, 2]:

$$
B_{c}=m_{\rho}^{2} / e \approx 10^{16} \text { Tesla, }
$$

where $m_{\rho}=775.5 \mathrm{MeV}$ is the mass of the $\rho$ meson. Such magnetic fields are expected to be experimentally reachable at the LHC [3].

When the strength of the uniform magnetic field is higher than critical (1.1) the usual vacuum of QCD is expected to experience a tachyonic instability which results in a formation of a new vacuum of the system. The main property of this new vacuum is a quark-antiquark condensate with quantum numbers of charged $\rho$-meson:

$$
\left\langle\bar{u} \gamma_{1} d\right\rangle=i\left\langle\bar{u} \gamma_{2} d\right\rangle=\rho\left(x_{\perp}\right) .
$$

Here $\rho\left(x_{\perp}\right)$ is a complex-valued function of (transverse) coordinates.

The condensing quark pairs are charged which implies that the vacuum becomes an electromagnetic superconductor. However, this new type of superconductivity has significantly different properties from conventional superconductors (see, for instance, [4]):

- Anisotropy: the ground state is a perfect conductor only for electrical fields parallel to magnetic fields. In transverse directions the vacuum is an insulator.

- Inhomogeneity: the condensate in ground state is a function of transverse coordinates and forms a lattice similar to the lattice of Abrikosov vortices in conventional superconductivity.

- Absence of Meissner effect: the magnetic field is not expelled from the superconductor.

- $\rho^{(0)}$-meson condensation: the condensation of charged $\rho$-meson induces the formation of neutral $\rho^{(0)}$-meson condensate [5].

Confirmations of this effect come from effective bosonic model [1], effective Nambu-JonaLasinio fermionic model [2], holographic approaches [6, 7] and numerical calculations [8]; there is an ongoing discussion of the problem in Refs. [9, 10, 11, 12].

\section{Vortex lattice ground state}

The condensate (1.2) can be described by one complex-valued function $\rho_{+}(x)$ which corresponds to charged $\rho$-mesons with the projection of spin $s_{z}=+1$ on the direction of the magnetic field:

$$
\rho_{+}(x)=\frac{1}{2} \bar{u} \gamma_{+} d, \quad \gamma_{+}=\gamma_{1}+i \gamma_{2}
$$

In $[1,2,5,7]$ the ground state of $\rho$-mesons for external magnetic fields slightly above critical $\left(\left|B-B_{c}\right| \ll B\right)$ was studied. It was found that the minimum energy configuration correponds to static, constant along magnetic field solution, which has in transverse direction a triangular periodic 


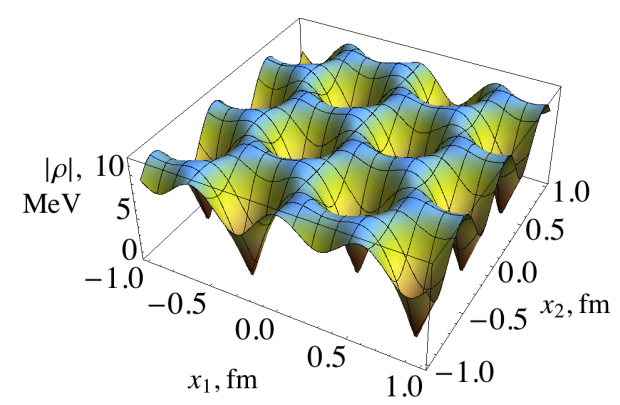

Absolute value of the condensate

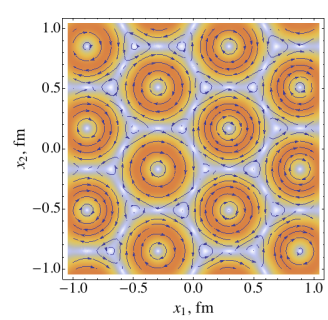

Superconducting currents

Figure 1: Lattice of $\rho$-vortices observed in the ground state in mean-field approximation. The figures are taken from [5]. The same structure is observed in holography [6].

structure (see Fig.1), resembling a lattice of Abrikosov vortices in conventional superconductivity. Similarly to Abrikosov vortices, when one goes around a zero of the condensate, the phase of the condensate changes by $2 \pi$. We call these new types of topological defects $\rho$-vortices.

The analytical study of [5] shows that the difference of energies between different types of lattices (triangular, square, etc.) is small, thus implying that vortices are weakly interacting. The quantum or thermal fluctuations can destroy the lattice and lead to formation of a liquid or a gas (see Fig.2).

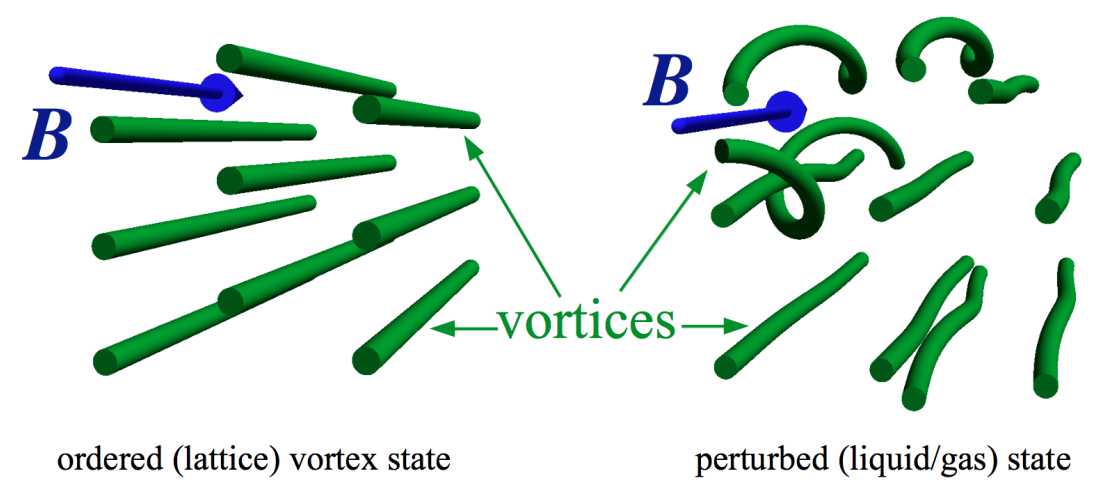

Figure 2: (left) The equilateral triangular vortex lattice in the mean-field approximation to the ground state of QCD and (right) its suggested melting due to the presence of quantum and/or thermal fluctuations.

\section{Numerical calculations}

In our numerical setup we basically follow Ref. [8]. We use lattice Monte-Carlo simulations of $S U$ (2) Yang-Mills lattice gauge theory. The quark fields are introduced by the overlap lattice Dirac operator $\mathscr{D}$ with exact chiral symmetry [13], and - due to the presence of the magnetic field - with twisted spatial boundary conditions [14]. The quarks are treated in the quenched approximation so that the vacuum quark loops are absent in our approach. We have studied $18^{4}$ and $19^{4}$ lattices in a wide range of the magnetic field strengths, $e B=(0 \ldots 2.14) \mathrm{GeV}^{2}$ with lattice spacings $a \approx 0.11 \mathrm{fm}$. We have used 20 gauge configurations per each value of the magnetic field. 
A direct study of the condensate (2.1) would be a confirmation of the existence of the superconducting phase. However we cannot measure the observable (2.1) in the quenched approximation. Instead we study the correlator of two operators (2.1):

$$
\phi(x)=\left\langle\rho_{+}(x) \rho_{+}^{\dagger}(0)\right\rangle_{A, B}=\operatorname{Tr}\left(\frac{1}{\mathscr{D}_{u}(A, B)+m} \gamma_{+} \frac{1}{\mathscr{D}_{d}(A, B)+m} \gamma_{+}^{\dagger}\right),
$$

Here subscripts $A, B$ denote that the correlator is measured in the fixed background of non-Abelian gauge field and external Abelian magnetic field.

The field $\phi(x)$ cannot be interpreted as a scalar charged field, because it is a correlator and falls off when the distance $x$ increases. To take into account this unwanted behaviour we normalize all observables by appropriate powers of $\phi$.

The observables associated with this new function $\phi$ are the following (appropriately discretized on the lattice):

- Normalized energy density $E(x)$

$$
E(x)=\frac{\left|D_{\mu} \phi(x)\right|^{2}}{|\phi(x)|^{2}}, \quad D_{\mu}=\partial_{\mu}-i e A_{\mu}
$$

- Normalized electric current $j_{\mu}(x)$

$$
j_{\mu}(x)=\frac{\phi^{*}(x) \vec{D}_{\mu} \phi(x)-\phi^{*}(x) \overleftarrow{D}_{\mu} \phi(x)}{2 i|\phi(x)|^{2}}
$$

- Local vortex density in transverse plane

$$
v(x)=\operatorname{sing} \arg \phi(x) \equiv \frac{\varepsilon^{a b}}{2 \pi} \frac{\partial}{\partial x_{a}} \frac{\partial}{\partial x_{b}} \arg \phi(x)
$$

We studied the energy density in two different planes - $(x, y)$ and $(x, z)$ - in order to find any signatures of $\rho$-vortices. Energy density is maximal in the center of $\rho$-vortex. Mean field approximation suggests that these vortices form a triangular lattice which is probably destroyed by quantum fluctuations. Thus we would expect emergence of some string-like defects aligned along the magnetic field described by large energy density. They would be seen as point-like defects in the energy density in the $(x, y)$ plane and as linelike structures in the $(x, z)$ plane.

Typical plots of energy density for three values of magnetic field - weak $\left(e B=0.356 \mathrm{GeV}^{2}\right)$, moderate $\left(e B=1.07 \mathrm{GeV}^{2}\right)$ and high $\left(e B=2.14 \mathrm{GeV}^{2}\right)$ are presented in Fig. 3. In accordance with our expectations at weak magnetic fields we did not see these structures, while at moderate magnetic fields we indeed observe string-like defects which are not however strictly ordered along magnetic field. At high magnetic fields the number of these structures grows, they become more parallel to the magnetic field and the whole structure exhibits properties of a melted vortex liquid.

In order to check whether these structures are physical we looked for other observables including vortex density (3.4) and (super)electromagnetic currents (3.3). We found that energy density and positions of vortices (3.4) are correlated and (super)currents encircle (3.3) the position of vortices (see Fig. 4). These observations allowed us to conclude that these vortices are indeed physical objects. 

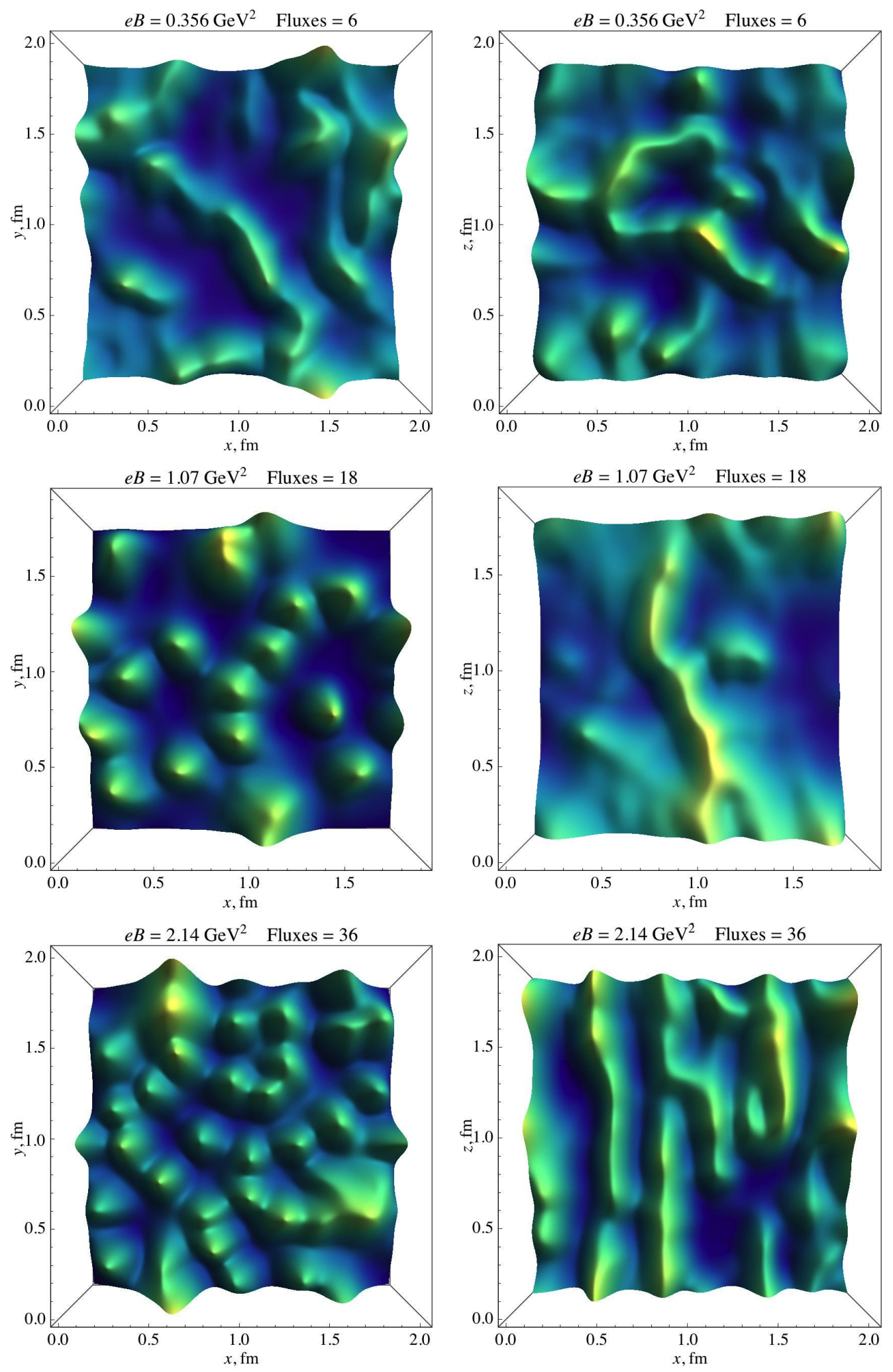

Figure 3: Typical behaviour of the energy density in the $(x, y)$ planes (the left column) and in the $(x, z)$ planes (the right column) for $e B=0.356 \mathrm{GeV}^{2}$ (the upper panel), $e B=1.07 \mathrm{GeV}^{2}$ (the middle panel) and $e B=2.14 \mathrm{GeV}^{2}$ (the lower panel). The number of elementary fluxes is $n=e B L_{x} L_{y} /(2 \pi)$. In the left column the magnetic field is perpendicular to the page and in the right column the magnetic field is directed vertically. 

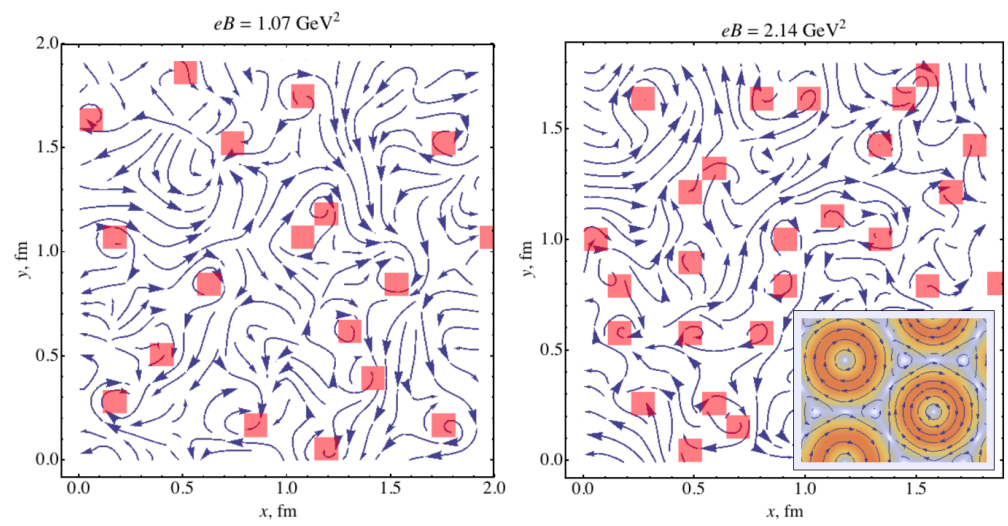

Figure 4: Examples of the superconducting currents (the blue lines), Eq. (3.3), around the vortices (the red squares), Eq. (3.4), in the $(x, y)$ planes at $e B=1.07 \mathrm{GeV}^{2}$ (left) and $e B=2.14 \mathrm{GeV}^{2}$ (right). According to the analytical expectations $[1,5]$ the currents should encircle the $\rho$ vortices in the clockwise direction (an example of the mean-field solution is shown in the inset of the right figure, from Ref. [5]).
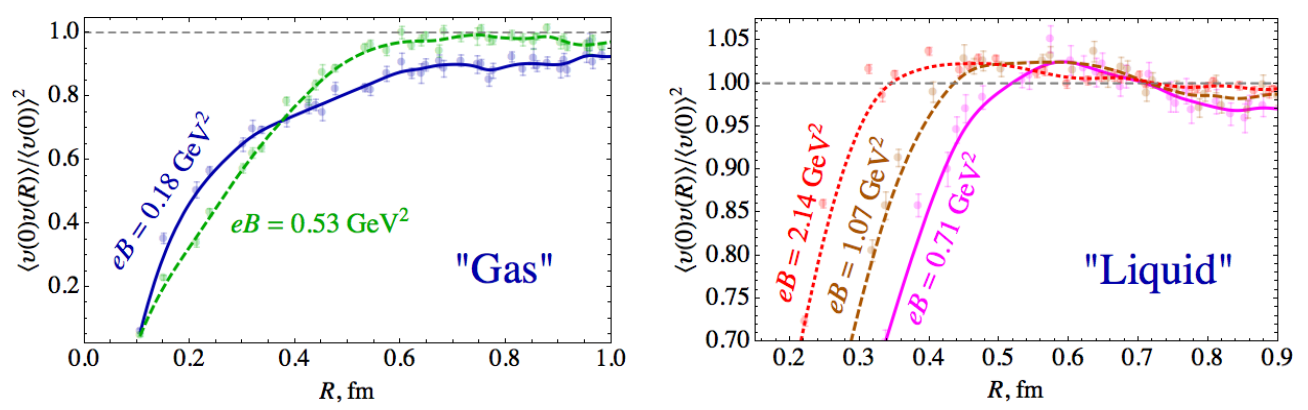

Figure 5: The normalised vortex-vortex correlation function $\langle v(0) v(R)\rangle /\langle v(0)\rangle^{2}$ in the $(x, y)$ plane. The monotonic (nonmonotonic) behaviour of the correlator signals the presence of the gas (liquid) vortex state at low (high) values of the magnetic field $B$ as shown in the left (right) plot.

Also to understand the structure of $\rho$-vortex state we studied two-vortices correlation function $g(R)=\langle v(0) v(R) /\rangle /\langle v(0)\rangle^{2}$, where $v(x)$ is a vortex density function (3.4). For low values of magnetic field the correlation function is a monotonically increasing function of $R$, implying that vortices form a (nonsuperconducting) gas of vortices (see Fig. 5 (left)). For high values of magnetic field this correlation function exhibits a rather wide peak at intermediate distance (see Fig. 5 (right)) which is an indication of formation a superconducting $\rho$-vortex liquid.

The melting of the vortex lattice in the quenched QCD might make it difficult to observe the suggested $\rho$-vortex condensation using the standard numerical tools (for details see [12]).

\section{Conclusions}

We have numerically observed the formation of the $\rho$-vortex liquid in the vacuum of quenched two-color QCD in strong magnetic field background. The vortex liquid phase is an electromagnetically superconducting phase characterised by the inhomogeneous order parameter (the $\rho$ meson condensate), similarly to the mixed (Abrikosov) phase of an ordinary type-II superconductor. In this phase the calculation of the (highly inhomogeneous) $\rho$-meson condensate by using the stan- 
dard methods may be unreliable. The transition between the usual (insulator) phase at low $B$ and the superconducting vortex liquid phase at high $B$ turns out to be very smooth.

\section{References}

[1] M. N. Chernodub, Superconductivity of QCD vacuum in strong magnetic field, Phys. Rev. D 82, 085011 (2010) [1008.1055].

[2] M. N. Chernodub, Spontaneous electromagnetic superconductivity of vacuum in strong magnetic field: evidence from the Nambu-Jona-Lasinio model, Phys. Rev. Lett. 106 (2011) 142003 [1101. 0117$].$

[3] A. Bzdak and V. Skokov, Event-by-event fluctuations of magnetic and electric fields in heavy ion collisions, Phys. Lett. B 710 (2012) 171 [1111.1949]; W.-T. Deng and X.-G. Huang, Event-by-event generation of electromagnetic fields in heavy-ion collisions, Phys. Rev. C 85 (2012) 044907 [1201.5108].

[4] M. N. Chernodub, Electromagnetic superconductivity of vacuum induced by strong magnetic field in Lect. Notes Phys. Strongly interacting matter in magnetic fields 871 (2013), edited by D. Kharzeev, K. Landsteiner, A. Schmitt, H.-U. Yee, [1208.5025]

[5] M. N. Chernodub, J. Van Doorsselaere and H. Verschelde, Electromagnetically superconducting phase of vacuum in strong magnetic field: structure of superconductor and superfluid vortex lattices in the ground state, Phys. Rev. D 85 (2012) 045002 [1111. 4401 ].

[6] N. Callebaut, D. Dudal and H. Verschelde, Holographic study of magnetically induced QCD effects, Acta Phys. Polon. Supp. 4 (2011) 671;

N. Callebaut, D. Dudal and H. Verschelde, Holographic rho mesons in an external magnetic field, JHEP 03 (2013) 033 [1105.2217];

M. Ammon, J. Erdmenger, P. Kerner and M. Strydom, Black Hole Instability Induced by a Magnetic Field, Phys. Lett. B 706 (2011) 94 [1106.4551].

[7] Y.-Y. Bu, J. Erdmenger, J. P. Shock and M. Strydom, Magnetic field induced lattice ground states from holography, JHEP 03 (2013) 165 [1210. 6669 ].

[8] V. V. Braguta et al, Electromagnetic superconductivity of vacuum induced by strong magnetic field: numerical evidence in lattice gauge theory, Phys. Lett. B 718 (2012) 667 [1104 . 3767].

[9] Y. Hidaka and A. Yamamoto, Charged vector mesons in a strong magnetic field, Phys. Rev. D 87 (2013) 094502 [1209.0007].

[10] M. N. Chernodub, Vafa-Witten theorem, vector meson condensates and magnetic-field-induced electromagnetic superconductivity of vacuum, Phys. Rev. D 86 (2012) 107703 [1209 . 3587 ].

[11] C. Li and Q. Wang, Amending the Vafa-Witten Theorem, Phys. Lett. B 721 (2013) 141 [1301. 7009 ].

[12] M. N. Chernodub, Comment on "Charged vector mesons in a strong magnetic field", (2013), 1309.4071.

[13] H. Neuberger, Exactly massless quarks on the lattice, Phys. Lett. B 417 (1998) 141 [hep-lat/9707022].

[14] M. H. Al-Hashimi and U. J. Wiese, Discrete Accidental Symmetry for a Particle in a Constant Magnetic Field on a Torus, Annals Phys. 324 (2009) 343. 